\title{
El Papel del Gerente y la Prospectiva en Escenarios de Crisis
}

\author{
The Role of the Manager and Foresight in Crisis Scenarios
}

\author{
Harold S. Lora-Guzmán \\ Universidad de Cartagena - Colombia \\ ORCID iD: https://orcid.org/0000-0001-6945-0999 \\ Hlorag@unicartagena.edu.co \\ Javier Prieto-Flórez* \\ Universidad de Cartagena - Colombia \\ ORCID iD: https://orcid.org/0000-0002-6885-2790 \\ javierprietoflorez@gmail.com
}

* Autor a quien debe ser dirigida la correspondencia
Fecha de recepción: 21/04/2021

Fecha de evaluación: 02/05/2021

Fecha de aceptación: 08/06/2021

Cómo citar: Lora-Guzmán, H., \& Prieto-Flórez, J. (2021). El papel del gerente y la prospectiva en escenarios de crisis. Revista Cientifica Anfibios, 4(1). https://doi.org/10.37979/afb.2021v4n1.86

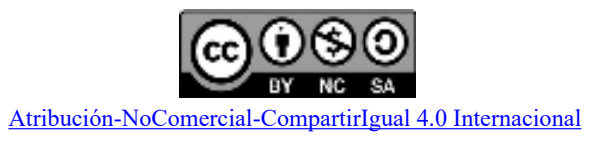

\section{Resumen}

El presente artículo expone el ejercicio del planeamiento estratégico y la prospectiva como una herramienta imprescindible en el ejercicio de la gerencia moderna esto dado por los escenarios de incertidumbre y complejidad que se vislumbran en los diferentes sectores económicos. Algunos aspectos que se consideran al estudiar estas incertidumbres son las dimensiones ambientales, económicas, sociales, políticas y aquellas intrínsecas asociadas a las propias características del ser humano. Lo expresado en este documentos forma parte de un trabajo de investigación que se viene realizando por los autores en cooperación con la cámara de comercio de Cartagena de indias y la gobernación de Bolívar ; en el que se pretende demostrar la importancia del estudio de los escenarios futuros que coadyuven a tomar mejores decisiones en el ejercicio de la toma de decisiones y la gerencia en PYMES; resalta la importancia de esta metodología y la utilización de alternativas estratégicas de carácter contingencial para definir políticas institucionales en momentos de crisis que coadyuvan al desarrollo económico y social de la región.

\section{Palabras clave}

Líder; manejador de conflictos; emprendedor; cambio; actores

\begin{abstract}
This article exposes the exercise of strategic planning and foresight as an essential tool in the exercise of modern management, given by the scenarios of uncertainty and complexity that can be seen in the different economic sectors. Some aspects that are considered when studying these uncertainties are the environmental, economic, social, political dimensions and those intrinsic associated with the characteristics of the human being. What is expressed in these documents is part of a research work that has been carried out by the authors in cooperation with the Chamber of Commerce of Cartagena de Indias and the government of Bolívar; In which it is intended to demonstrate the importance of studying future scenarios that contribute to making better decisions in the exercise of decision-making and management in SMEs; highlights the importance of this methodology and the use of contingent strategic alternatives to define institutional policies in times of crisis that contribute to the economic and social development of the region.
\end{abstract}




\section{Keywords}

Leader; conflict manager; entrepreneur; change; stakeholders

\section{Introducción}

A nivel mundial los cambios drásticos que se presentan en los diferentes sectores económicos llevan a las empresas a buscar diferentes herramientas que coadyuven al análisis de información, protección, organización, conversión y análisis de esta en datos e inteligencia para facilitar la toma de decisiones lo que se ha convertido en una herramienta fundamental en la gerencia moderna (Solleiro \& Castañón 1998; Fram \& Licona, 2016). Gutiérrez (2006) considera que además del planeamiento estratégico, el análisis, estudio e interpretación de los escenarios futuros es una herramienta básica para el ámbito de estudio del entorno organizacional, y una puesta en escena de una dimensión del conocimiento que hace parte del ejercicio gerencial y la toma de decisiones.

Con una visión holística de la definición de prospectiva se aborda la planeación de escenarios que decanta en la creación de políticas públicas y empresariales que contribuyan al desarrollo económico y social de las regiones, así como la pertinencia de estrategias racionales y contingenciales que emergen de la elaboración dicha política, definiendo el alcance de objetivos a mediano y largo plazo. Se pretende que la gerencia y administración de las PYMES conozca y defina una metodología que facilite la construcción de estrategias y escenarios futuribles, dando como resultado la utilización de una herramienta de análisis y toma de decisiones para la generación de políticas en los diferentes niveles de este tipo de organizaciones. Este trabajo forma parte de un trabajo de investigación en curso apoyado por la gobernación de Bolívar y la cámara de comercio de Cartagena (CCC) que pretende coadyuvar a la empleabilidad, el desarrollo económico y social a través del impulso al sector PYME

\section{Marco teórico}

\section{Gerencia estratégica y prospectiva. Conceptos y evolución}

La estrategia como concepto proviene del griego strategos (general). El termino se utilizó por varias décadas en los ejércitos, milicias y fuerzas armadas en el contexto de los planes, ventajas que se desconocían por parte del ejercito enemigo y eran utilizadas para poder derrotarlos (Weihrich y Koontz, 1993). La mayor referencia a lo anteriormente expuesto se puede observar en el libro "El arte de la guerra" de Sun Tzu 400-340 (A.C.) que es referente obligado de todo aquel que se considere interesado en el aprendizaje del pensamiento estratégico y la estrategia. En ese mismo sentido, Codina (2006) al hacer referencia a la importancia de la obra de Sun Tzu determina que, en los tratados modernos asociados a la temática de negocios, gerencia, empresa se utilizan los mismos vocablos del texto enmarcados en la diatriba de la concepción actual como por ejemplo enemigo y competencia.

Para Tarzijan (2008), el devenir histórico y el desarrollo del planeamiento estratégico se puede estudiar en razón a cuatro etapas fundamentales. La primera que data de 1920 y se le considera "Planificación financiera", la segunda etapa se asocia a las diferentes estrategias con las que se pretende garantizar "la planificación financiera a largo plazo", la tercera etapa se enmarca en el análisis global de la empresa y su entorno abarcando una estrategia unificada teniendo en cuenta todas las áreas de la organización. En esta etapa aparecen autores de alto referente académico tales como Ansoff, Andrews, Drucker y Chandler.

Tarziján, (2008) considera que la última etapa tiene como característica primordial el conocimiento y aprendizaje organizacional y el estudio del entorno en el que se desarrollan los negocios, es decir, el entorno y la posición competitiva. Este análisis del entorno económico inicia con una etapa de diagnóstico de la situación actual en la empresa desde una perspectiva interna y externa, evaluando las fortalezas y debilidades y las oportunidades y amenazas respectivamente. En esta etapa aparecen autores que revisten gran importancia por la creación de una serie de herramientas metodológicas e instrumentos que permiten el desarrollo de diagnósticos y la generación de estrategias alternativas entre los cuales podemos resaltar: Michael Porter (modelo de las cinco fuerzas), 
Andrews (Matriz FODA), Ansoff (Matriz Ansoff), Chandler y Drucker entre otros.

Con respecto al estudio de la prospectiva, el interés por el análisis, aplicación y estudio del tema se ha incrementado en las últimas décadas. Para su análisis y comprensión existen dos grandes corrientes la norteamericana y la francesa. En cuanto a la escuela francesa presenta mayor influencia y expansión en Europa y América latina afianzando sus estudios y utilización en lo que se conoce como la prospectiva estratégica; para el caso de la escuela norteamericana con influencia en el mundo anglosajón analiza los estudios y desarrollo de teorías a través de la futurología.

Se considera que la tradición francesa en cuanto a sus inicios se establece luego de la segunda guerra mundial. Presenta este movimiento gran influencia en el sentido social cuyo exponente principal se le considera a Gastón Berger, expandiendo su visión política teniendo como base los estudios de Bertrand de jouvenel. Este autor considera que la denominación de futurible abarca tres aspectos importantes decisión, libertad y poder elementos importantes para el enfoque prospectivo (de Jouvenel, 2004). Ahora bien, para el caso de la escuela norteamericana se fundamenta en el forecasting o predicción desarrollando escenarios futuros basándose en modelos matemáticos y herramientas estadísticas tales como la regresión lineal por el método de los mínimos cuadrados. Esta nueva dinámica presenta expositores que desarrollan metodologías nuevas para el uso de la prospectiva tales como rueda de futuros, método Delphi, método del análisis de impactos cruzados. (Randers, 2012)

El concepto de prospectiva desde la visión de la escuela francesa considera que las probabilidades que se pueden establecer en un diagnóstico inicial solo permiten "disminuir la incertidumbre" (Godet 1985), esto teniendo en cuenta que se había rechazado previamente el determinismo en razón al futuro que lo considera amplio y variable. Mojica (2011:139) de igual manera establece que el ejercicio de la prospectiva "no espera establecer parámetros sobre eventos futuros por el contrario considera llegar a él a través de las conjeturas". Godet y Durance (2009: 9), plantean que "en el desarrollo de la prospectiva, es de vital importancia el futuro dado que se encuentra en proceso de construcción por actores interesados en el éxito y viabilidad de sus proyectos.

\section{La estrategia y herramientas para la gerencia estratégica}

Ante situaciones de incertidumbre en los mercados y sectores económicos, las organizaciones apuestan por el desarrollo y puesta en marcha de estrategias que les permitan a las organizaciones afrontar los cambios mediante el desarrollo de tecnologías de comunicación y la puesta en marcha de estudios prospectivos (Del Río-Cortina, 2021). Con respecto al concepto de estrategia, Henry Mintzberg la define como "la guía o modelo que agrupa los principales objetivos, metas y políticas de una empresa, y a su vez define la forma coherente en que se deben realizar las acciones para darle cumplimiento a lo propuesto" (Labarca, 2008). Para una mejor comprensión del significado Mintzberg divide el termino en cinco definiciones a saber estrategia como patrón, como posición, como plan, como pauta de acción y como perspectiva.

Briola (2007), teniendo como referente a Mintzberg, amplia los conceptos así: al utilizar la estrategia como plan, se le reconoce como el procedimiento, los pasos consientes para poder abordar una situación en particular. La utilización de la estrategia como pauta de acción implica un movimiento para triunfar ante la competencia, una estrategia desarrollada como patrón señala el comportamiento que se debe asumir en el entorno competitivo; la estrategia como posición define la posición actual de la organización en el mercado que compite y por último la estrategia como perspectiva, se considera la lectura que la organización hace del entorno competitivo donde se desarrolla. Mintzberg, Ahlstrand y Lampel (2003) señalan que concurren diez escuelas que clasifican las estrategias en dos grandes grupos: escuelas prescriptivas y descriptivas.

En el primer grupo encontramos las escuelas de diseño, planificación y posicionamiento que tienen como propuesta de estudio la formulación de la estrategia y fusionadas, definen lo que se conoce como el pensamiento estratégico racional, estableciendo el concepto conocido como la formulación estratégica de carácter normativo. En el segundo grupo, encontramos escuelas tales como integra- 
dora, política, del aprendizaje y del entorno que analizan y demuestran cómo se construyen y de donde sales las estrategias en las empresas.

A continuación, presentaremos el modelo de direccionamiento estratégico más utilizado desde la óptica de sus autores, y luego se presentarán algunas de las herramientas que se utilizan para la construcción de estrategias alternativas en las organizaciones luego de haber desarrollado el diagnóstico de la situación actual.

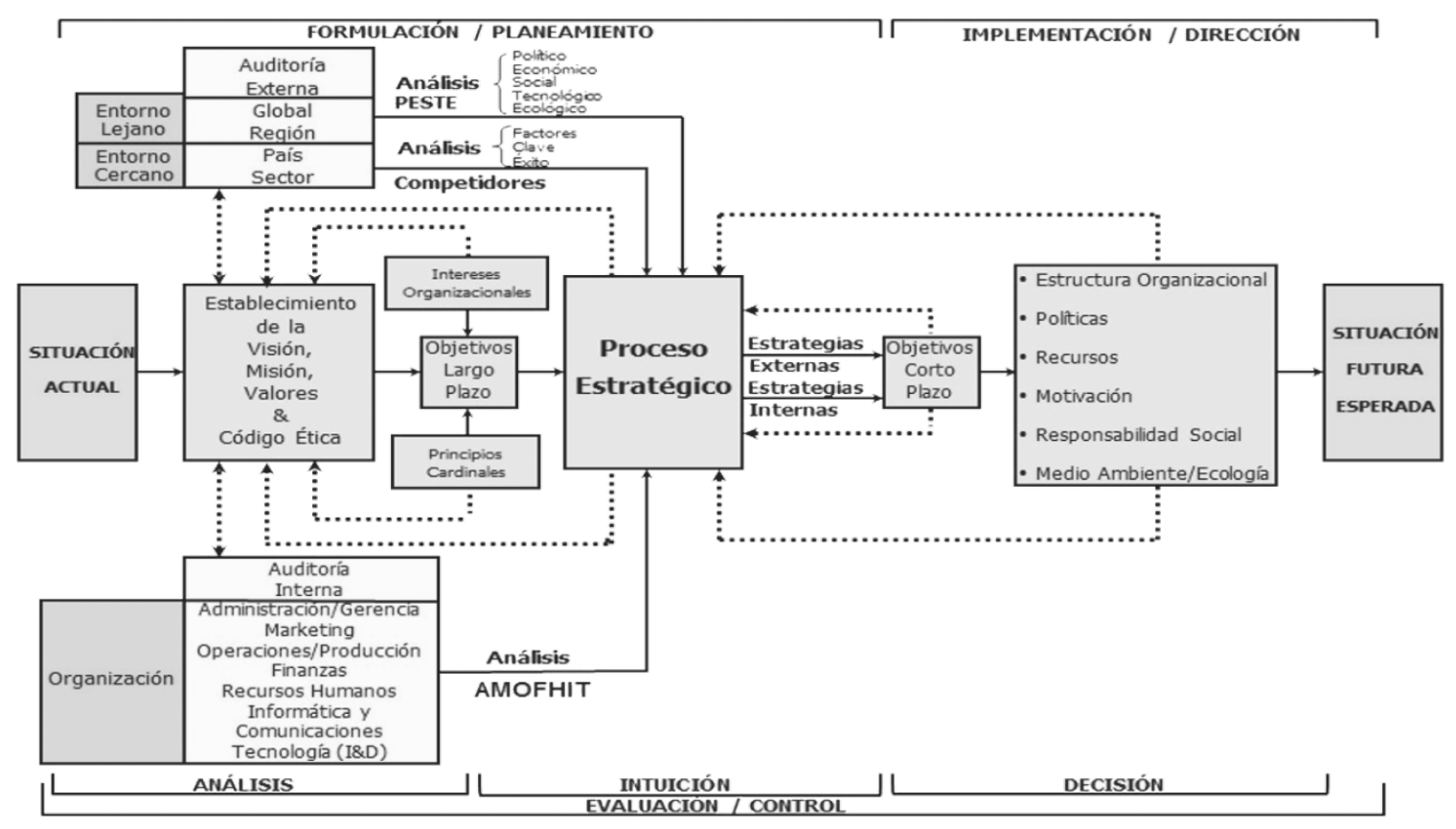

Figura 1. Modelos secuencial del proceso estratégico Fuente: tomado del proceso estrtégico: un enfoque de gerencia D' Alessio (2013)

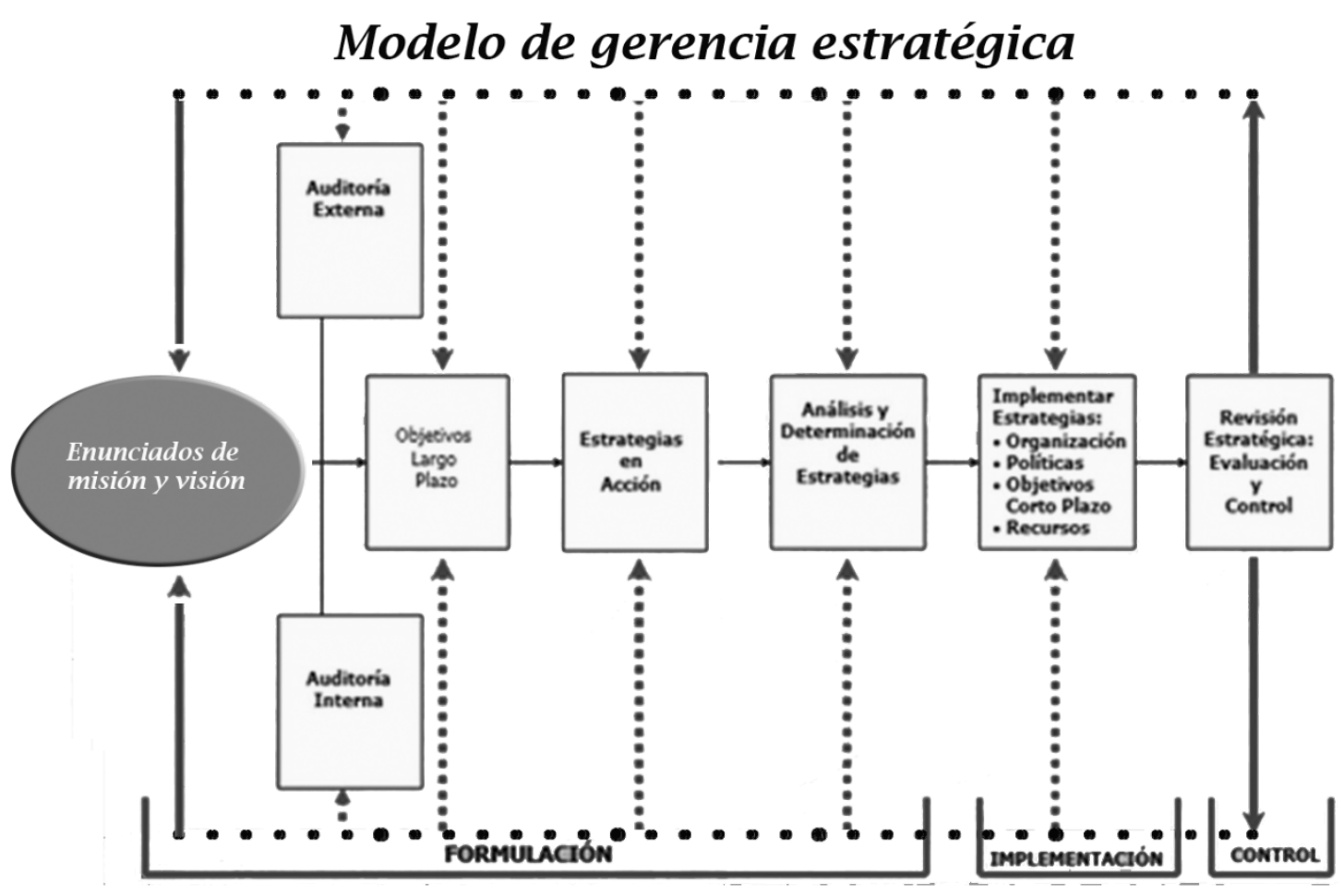

Figura 2. Modelo de gerencia estratégica Davies (2013).

Fuente: conceptos de administración estratégica, Pág. 132. 


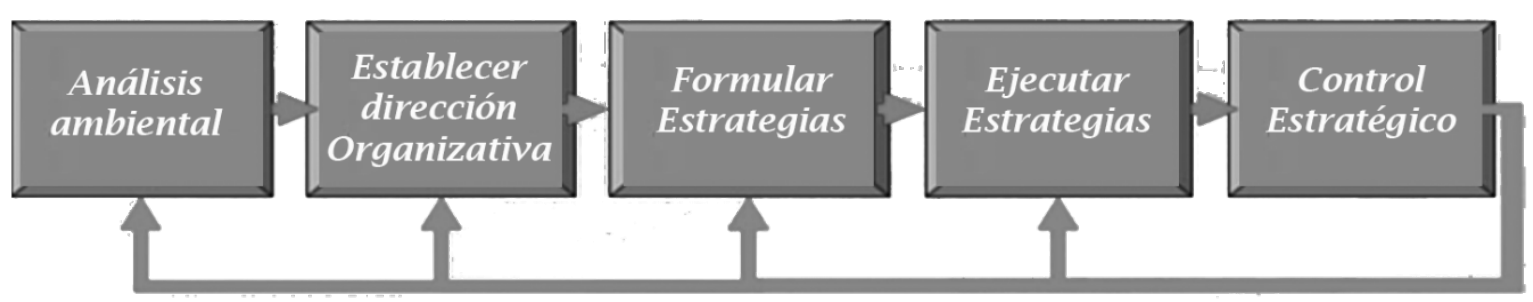

\section{Retroalimentación}

Figura 3. Proceso de administración estratégica

Fuente: Modern management: concepts and skills (Certo, 2008)

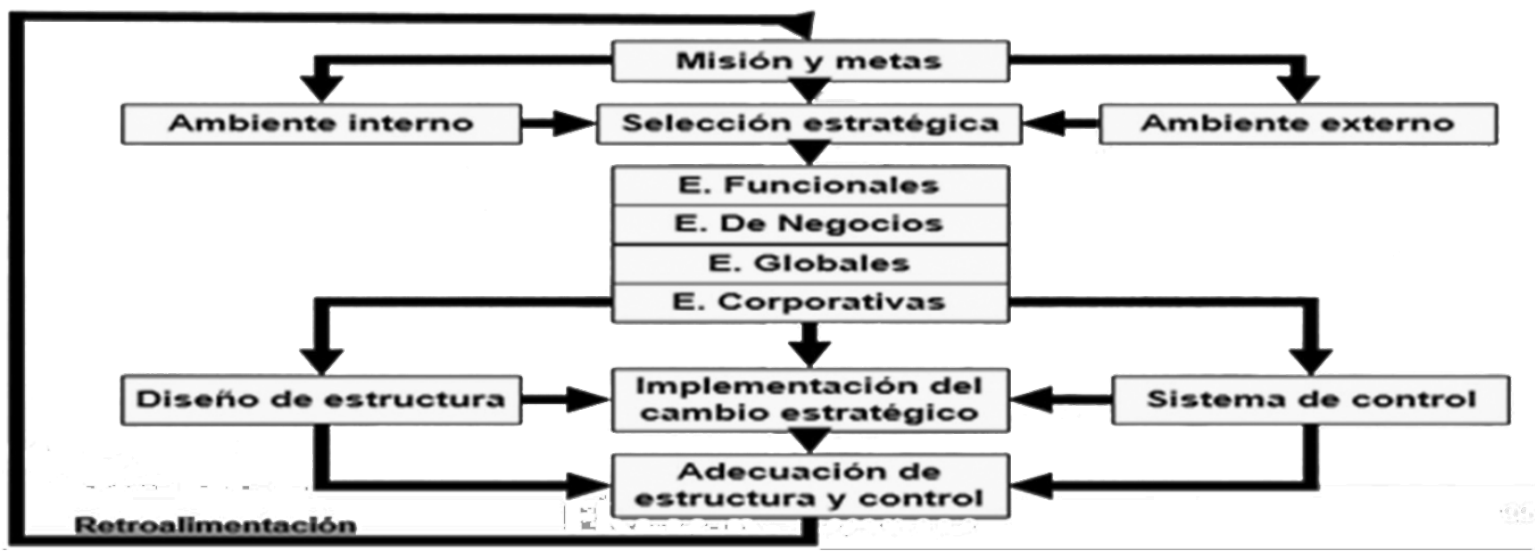

Figura 4. Modelo de Hill-Jones.

Fuente: Administración estratégica (Hill et al., 2019)

\section{Metodología}

Los modelos que se relacionan establecen la metodología para el direccionamiento estratégico, compuesto principalmente por tres etapas o fases la formulación, implementación y control. Inicialmente se establece un análisis de la situación actual, seguido por la definición de la filosofía institucional visión, la misión, los valores, políticas y el código de ética; lo que se utilizara como guía y norma para el accionar de la empresa. Posteriormente se analiza en la fase de diagnóstico la industria de manera global y las características intrínsecas de la organización a través de su análisis interno. Definidos los objetivos de la organización y las políticas que servirán como base para lograrlos, se debe iniciar el análisis de lo que se denomina posición competitiva relativa a través de la utilización de matrices tales como PESTEL, el modelo de las cinco fuerzas de Porter, el análisis de los productos y mercados a través de la matriz de Ansoff, la matriz MEFE y la matriz POAM (perfil oportunidades y amenazas del medio), MPC (matriz del perfil competitivo) y MPR (matriz del perfil referencial)
Dada la utilización de las matrices referenciadas, se puede realizar la evaluación externa que permite conocer elementos del entorno tales como oportunidades y amenazas clave, la posición actual de la competencia y aquellos factores que son requisito sine qua non para la permanencia y futuro de las organizaciones, los Factores Críticos de Éxito (FCE), que coadyuvan a la gerencia en la formulación de estrategias y lograr ventajas competitivas con el aprovechamiento de las oportunidades, evitar y/o reducir el impacto de las amenazas Saavedra, (2005).

\section{Resultados}

\section{PEST}

Los insumos utilizados por esta matriz son principalmente el análisis de los competidores, los datos estadísticos de los organismos de gobierno, estudios de gremios, investigaciones de rigor académico entre otros. La metodología de aplicación del análisis PEST consiste en la 
compilar información para su análisis y a tra- lleres con expertos, utilizando técnicas como vés de diversas técnicas realizar debate sobre Brainstorming y método Delphi.

los datos ejemplo investigación de campo, Ta-

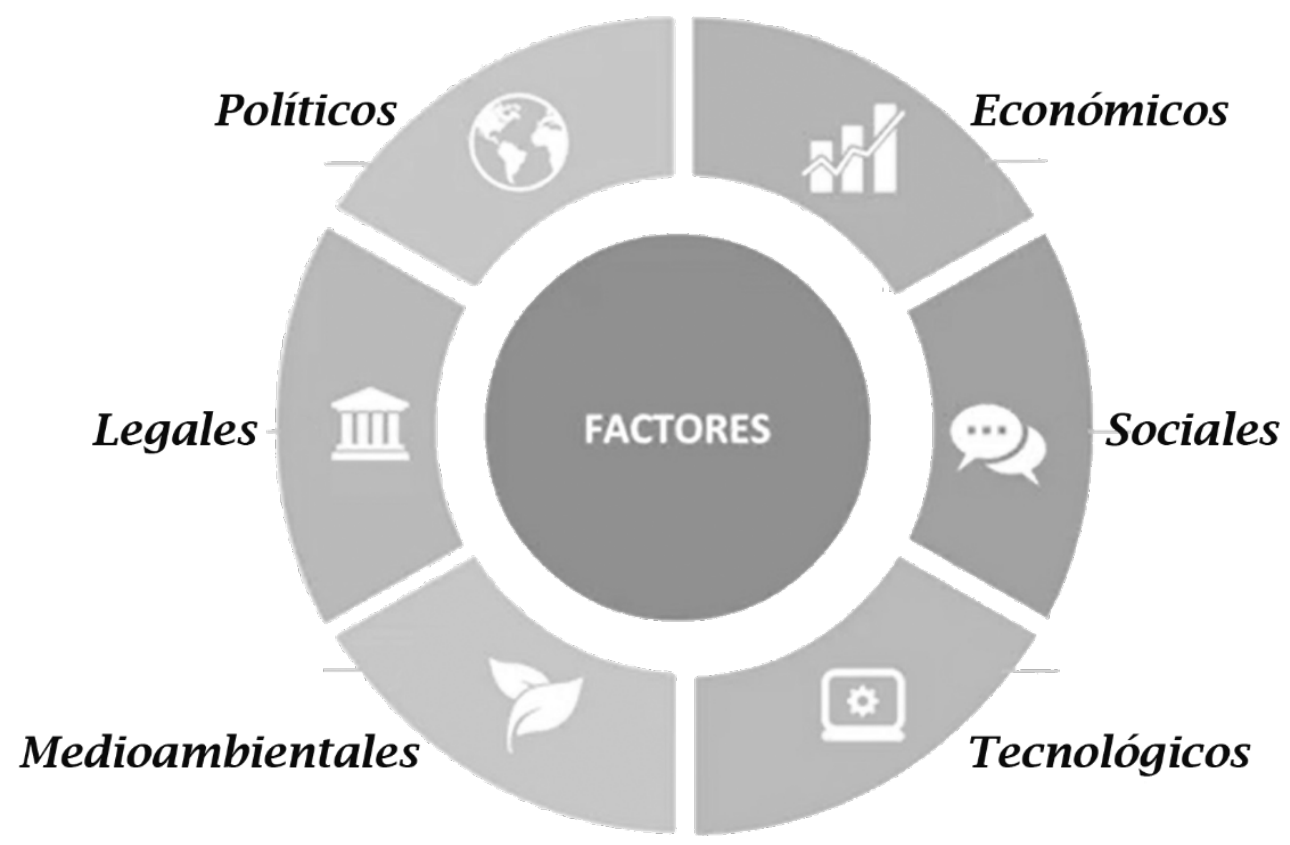

Figura 5. Factores a tener en cuenta para la construcción de matriz PESTEL Fuente: elaboración propia

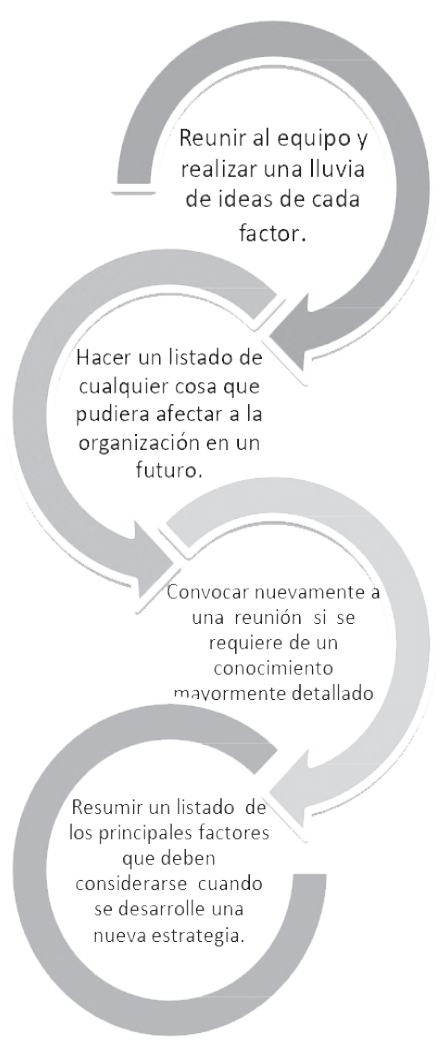

Figura 6. Como hacer un análisis PESTEL

Fuente: elaboración propia 


\section{Modelo de Porter}

Uno de los modelos más conocidos en el análisis del entorno, desarrollado por Michael Porter (Estrategia Competitiva, CECSA). El autor considera que existen cinco fuerzas que intervienen en el mercado: la amenaza de nuevos competidores, rivalidad entre competidores, amenaza de productos sustitutos, el poder de negociación de los clientes y el poder de negociación de los proveedores.

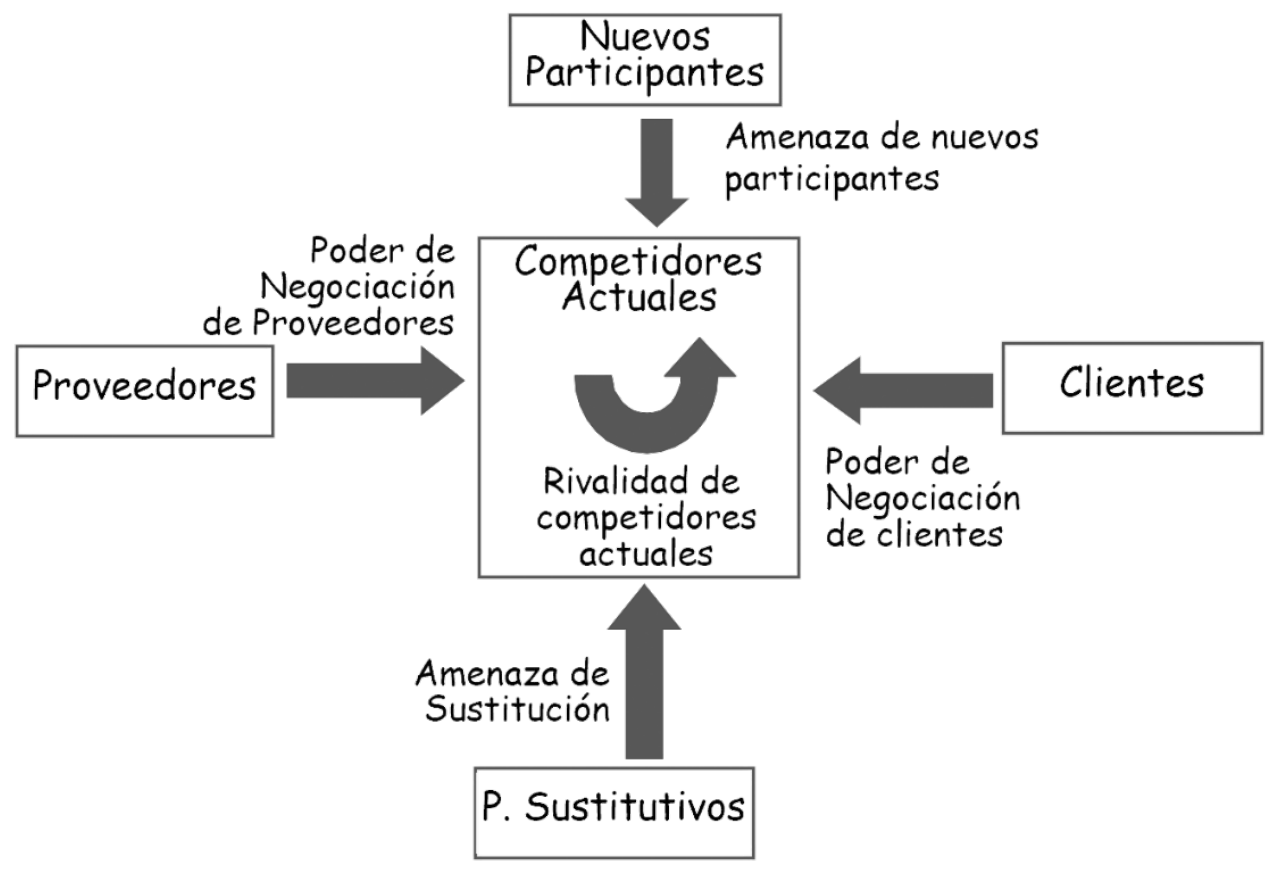

Figura 7. Las cinco fuerzas de Porter Fuente: Porter (2008)

\section{MEFE}

Suele ser muy implementada para el análisis y evaluación de los factores económico, social, cultural, demográfico, ambiental, política, gubernamental, legal, tecnológica y competitiva. (David, 2013)

Tabla 1. Matriz MEFE

\begin{tabular}{lrrr}
\hline $\begin{array}{l}\text { UST-Factores Externos Clave } \\
\text { Oportunidades }\end{array}$ & Peso & Valor & Puntaje Ponderado \\
\hline Mercados globales sin cubrir & 0,15 & 1 & 0,15 \\
Mayor demanda & 0,05 & 3 & 0,15 \\
Desmesurada expansión de Internet & 0,05 & 1 & 0,05 \\
Pinkerton líder del mercado de descuentos & 0,15 & 4 & 0,6 \\
Más presión social para dejar de fumar & 0,1 & 3 & 0,3 \\
Amenazas & & & 0,1 \\
Legislación contra la industria tabacalera & 2 & 0,15 \\
Límites a la producción de tabaco & 0,05 & 3 & 0,1 \\
Mercado sin humo: región SE de U.S.A. & 0,05 & 2 & 0,2 \\
Mala exposición de los medios del FDA & 0,1 & 2 & 0,2 \\
Administración de Clinton & 0,2 & 1 & 2,1 \\
TOTAL & 1 & & 0.2 \\
\hline \hline
\end{tabular}


MPC

Esta matriz identifica las empresas que se consideren los principales competidores de la misma; analizando sus fortalezas y debilidades de acuerdo a una firma que se toma como referen- te. En la MPC las calificaciones y las puntuaciones ponderadas totales para las compañías rivales pueden ser comparadas contra la compañía muestra. Este análisis comparativo arroja importante información estratégica interna. (David, 2013)

Tabla 2. Matriz MEFE

\begin{tabular}{|c|c|c|c|c|c|c|c|c|}
\hline (MPC) & & & & & & B & & $\mathrm{C}$ \\
\hline Factor determinante de éxito & Peso & Valor & & Puntaje & Valor & Puntaje & Valor & Puntaje \\
\hline Publicidad & 0,2 & & 1 & 0,2 & 4 & 0,8 & 3 & 0,6 \\
\hline Calidad de Producto & 0,1 & & 4 & 0,4 & 4 & 0,4 & 3 & 0,3 \\
\hline Competencia de precios & 0,1 & & 3 & 0,3 & 3 & 0,3 & 4 & 0,4 \\
\hline Gestión & 0,1 & & 4 & 0,4 & 3 & 0,3 & 3 & 0,3 \\
\hline Posición Financiera & 0,15 & & 4 & 0,6 & 3 & 0,45 & 3 & 0,45 \\
\hline Lealtad del Cliente & 0,1 & & 4 & 0,4 & 4 & 0,4 & 2 & 0,2 \\
\hline Expansión global & 0,2 & & 4 & 0,8 & 2 & 0,4 & 2 & 0,4 \\
\hline Participación de mercado & 0,05 & & 1 & 0,05 & 4 & 0,2 & 3 & 0,15 \\
\hline Total & 1 & & & 3,15 & & 3,25 & & 2,8 \\
\hline
\end{tabular}

Valores: 4. Fortaleza mayor, 3. Fortaleza menor, 2. Debilidad Menor, 1. Debilidad mayor

Fuente: Conceptos de administración estratégica David (2013)

\section{Matriz Ansoff}

El autor considera que el objetivo principal de toda organización es la mejora de la rentabilidad ante lo anterior, se presentan tres posibilidades contraerse, mantenerse y optar por el crecimiento. Ante estas tres posibilidades la organización necesita la siguiente información: cual es la posición actual de la empresa, que posicionamiento quiere alcanzar en el futuro y cuál sería el camino adecuado para cumplir esa meta. Para Ansoff, inicialmente se debe definir el concepto del negocio, para lo anterior es imprescindible definir cuáles son sus productos-mercados. La idea de acuerdo al autor es encontrar un "vínculo común" entre productos y mercados logrando un seguimiento de los mismos y por ende mayor control.

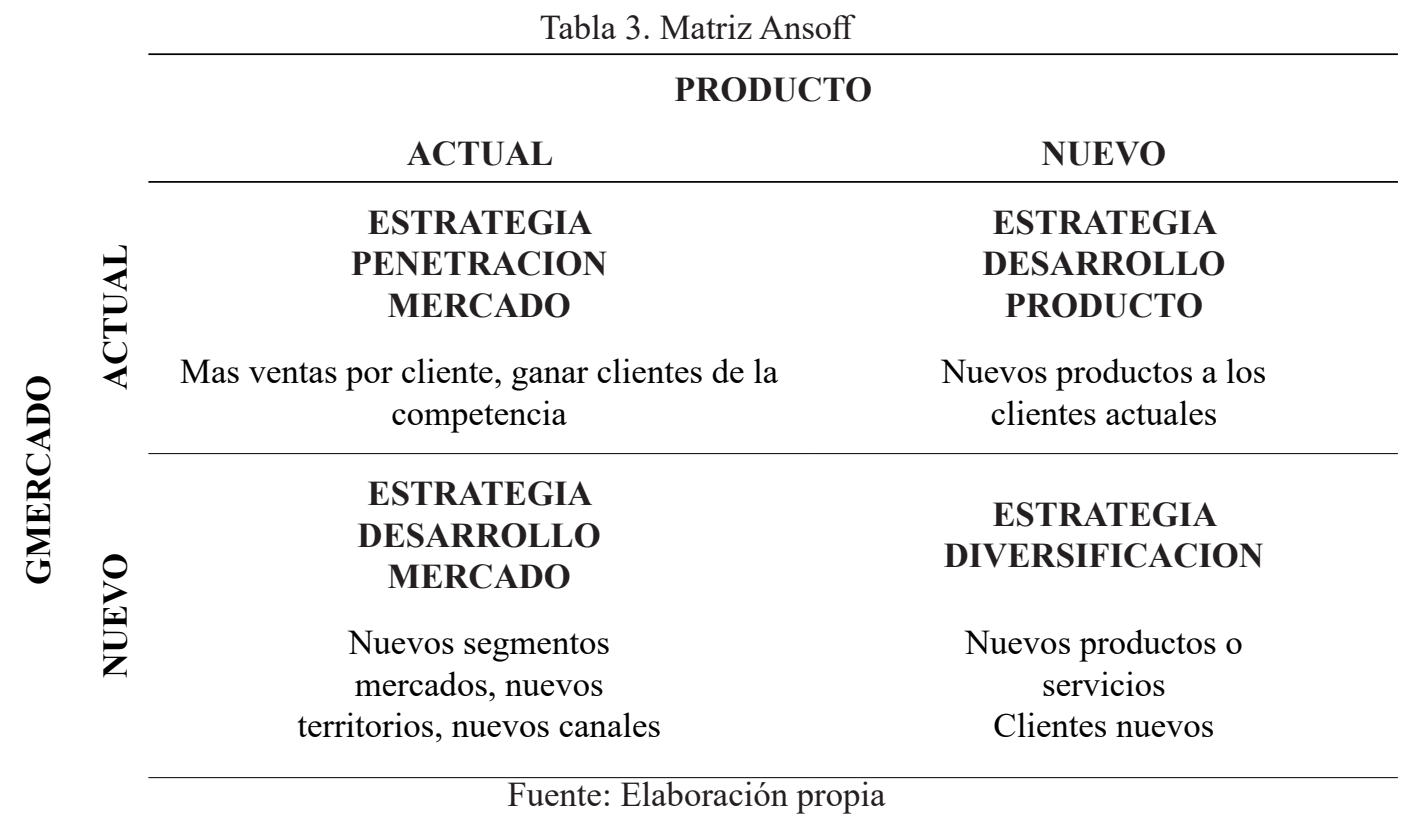


La matriz perfil oportunidades y amenazas del medio (POAM)

Es una matriz utilizada para la identificación y valoración de amenazas y oportunidades con ca- rácter potencial para la empresa. Esta valoración se realiza dependiendo de su impacto e importancia. La matriz evalúa 5 factores a considerar: Factor económico, tecnológico, competitivo, político y social. Serna (2008)

Tabla 4. Matriz POAM

ANÁLISIS EXTERNO

OPORTUNIDADES AMENAZAS IMPACTO

\begin{tabular}{|c|c|c|c|c|c|c|c|c|c|c|}
\hline & & $\mathbf{A}$ & $\mathbf{M}$ & $\mathbf{B}$ & $\mathbf{A}$ & $\mathbf{M}$ & B & $\mathbf{A}$ & $\mathbf{M}$ & B \\
\hline \multirow{11}{*}{$\begin{array}{l}\text { FACTORES SOCIA- } \\
\text { LES }\end{array}$} & Poder de compra de los clientes. & & & & & & $\mathrm{X}$ & & & $\mathrm{X}$ \\
\hline & Valores culturales. & $\mathrm{X}$ & & & & & & & $\mathrm{X}$ & \\
\hline & Cultura social. & & & & $\mathrm{X}$ & & & $\mathrm{X}$ & & \\
\hline & Turismo cultural & $\mathrm{X}$ & & & & & & $\mathrm{X}$ & & \\
\hline & Variaciones demográficas. & & $\mathrm{X}$ & & & & & $\mathrm{X}$ & & \\
\hline & Medios de Comunicación. & & $\mathrm{X}$ & & & & & $\mathrm{X}$ & & \\
\hline & Reglamentación social. & $\mathrm{X}$ & & & & & & $\mathrm{X}$ & & \\
\hline & Nivel educativo poblacional. & & & $\mathrm{X}$ & & & & & $\mathrm{X}$ & \\
\hline & Bajos ingresos de la población. & & & & $\mathrm{X}$ & & & $\mathrm{X}$ & & \\
\hline & Movilizaciones sociales & & & & $\mathrm{X}$ & & & $\mathrm{X}$ & & \\
\hline & Inseguridad social. & & & & $\mathrm{X}$ & & & $\mathrm{X}$ & & \\
\hline \multirow{7}{*}{$\begin{array}{l}\text { FACTORES } \\
\text { ECONOMICOS }\end{array}$} & $\begin{array}{l}\text { Disponibilidad de líneas de cré- } \\
\text { dito. }\end{array}$ & & $\mathrm{X}$ & & & & & & $\mathrm{X}$ & \\
\hline & Poder adquisitivo del cliente. & & & & $\mathrm{X}$ & & & $\mathrm{X}$ & & \\
\hline & Inversión social. & & $\mathrm{X}$ & & & & & & $\mathrm{X}$ & \\
\hline & $\begin{array}{l}\text { Crecimiento de la economía na- } \\
\text { cional. }\end{array}$ & & & & & $\mathrm{X}$ & & & $\mathrm{X}$ & \\
\hline & Acceso a combustible & & & & $\mathrm{X}$ & & & $\mathrm{X}$ & & \\
\hline & Inflación & & & & $\mathrm{X}$ & & & $\mathrm{X}$ & & \\
\hline & Creación de Nuevos Impuestos. & & & & $\mathrm{X}$ & & & $\mathrm{X}$ & & \\
\hline \multirow{4}{*}{$\begin{array}{c}\text { FACTORES } \\
\text { COMPETITIVOS }\end{array}$} & Estrategia de los competidores. & & & & $\mathrm{X}$ & & & $\mathrm{X}$ & & \\
\hline & Competencia desleal. & & & & $\mathrm{X}$ & & & $\mathrm{X}$ & & \\
\hline & Nuevos competidores. & & & & & $\mathrm{X}$ & & & $\mathrm{X}$ & \\
\hline & Alianzas estratégicas. & & & & & $\mathrm{X}$ & & & $\mathrm{X}$ & \\
\hline $\begin{array}{c}\text { FACTORES } \\
\text { TECNOLOGICOS }\end{array}$ & Acceso a tecnología de Punta. & $\mathrm{X}$ & & & & & & $\mathrm{X}$ & & \\
\hline \multirow{3}{*}{$\begin{array}{c}\text { FACTORES } \\
\text { GEOGRARCOS }\end{array}$} & Cambio Climático. & & & & & $\mathrm{X}$ & & $\mathrm{X}$ & & \\
\hline & Vías de Acceso. & & & & $\mathrm{X}$ & & & $\mathrm{X}$ & & \\
\hline & Fenómenos Naturales. & & & & & $\mathrm{X}$ & & $\mathrm{X}$ & & \\
\hline
\end{tabular}

Fuente: Gerencia estratégica Serna (2008)

\section{Análisis interno}

Como complemento de lo anterior, se debe revisar al interior de la organización como puede dar respuesta al entorno externo revisando sus fortalezas y debilidades. En razón a lo anterior revisaremos las matrices para la revisión interna.

\section{FODA}

Consiste en el análisis de los elementos internos que se consideren como fortalezas y debilidades, que al revisar cada uno de los departamentos y áreas dan a conocer la situación interna de la organización. El proceso es sencillo y por medio del mismo se obtiene una perspectiva global de 
la situación actual de una empresa determinada. momento actual y a través de ella se ajusta la caThompson y Strikland (1998) consideran que pacidad interna de la empresa con respecto a una FODA estima el resultado de la estrategia en el lectura previa del entorno.

Tabla 5. Matriz DOFA

\begin{tabular}{|c|c|}
\hline FORT & DEBILIDADES \\
\hline $\begin{array}{l}\text { Capacidades fundamentales en áreas claves. } \\
\text { Recursos financieros adecuados. } \\
\text { Buena imagen de los compradores. } \\
\text { Ser un reconocido líder en el mercado. } \\
\text { Estrategias de las áreas funcionales bien ideadas. } \\
\text { Acceso a economías de escala. } \\
\text { Aislada (por lo menos hasta cierto grado) de las fuertes } \\
\text { presiones competitivas. } \\
\text { Propiedad de la tecnología. } \\
\text { Ventajas en costos. } \\
\text { Mejores campañas de publicidad. } \\
\text { Habilidades para la innovación de productos. } \\
\text { Dirección capaz. } \\
\text { Posición ventajosa en la curva de experiencia. } \\
\text { Mejor capacidad de fabricación. } \\
\text { Habilidades tecnológicas superiores. }\end{array}$ & $\begin{array}{l}\text { No hay una dirección estratégica clara. } \\
\text { Instalaciones obsoletas. } \\
\text { Rentabilidad inferior al promedio. } \\
\text { Falta de oportunidad y talento gerencial. } \\
\text { Seguimiento deficiente al implantar la estrategia. } \\
\text { Abundancia de problemas operativos internos. } \\
\text { Atraso en investigación y desarrollo. } \\
\text { Línea de productos demasiado limitada. } \\
\text { Débil imagen en el mercado. } \\
\text { Débil red de distribución. } \\
\text { Habilidades de mercadotecnia por debajo del pro- } \\
\text { medio. } \\
\text { Incapacidad de financiar los cambios necesarios en } \\
\text { la estrategia. } \\
\text { Costos unitarios generales más altos en relación } \\
\text { con los competidores clave. }\end{array}$ \\
\hline & \\
\hline $\begin{array}{l}\text { Atender a grupos adicionales de clientes. } \\
\text { Ingresar en nuevos mercados o segmentos. } \\
\text { Expandir la línea de productos para satisfacer una } \\
\text { gama mayor de necesidades de los clientes. } \\
\text { Diversificarse en productos relacionados. } \\
\text { Integración vertical (hacia adelante o hacia atrás). } \\
\text { Eliminación de barreras comerciales en mercados forá- } \\
\text { neos atractivos. } \\
\text { Complacencia entre las compañías rivales. } \\
\text { Crecimiento más rápido en el mercado. }\end{array}$ & $\begin{array}{l}\text { Entrada de competidores foráneos con costos me- } \\
\text { nores. } \\
\text { Incremento en las ventas y productos sustitutos. } \\
\text { Crecimiento más lento en el mercado. } \\
\text { Cambios adversos en los tipos de cambio y políti- } \\
\text { cas comerciales de gobiernos extranjeros. } \\
\text { Requisitos reglamentarios costosos. } \\
\text { Vulnerabilidad a la recesión y ciclo empresarial. } \\
\text { Creciente poder de negociación de clientes o pro- } \\
\text { veedores. } \\
\text { Cambio en las necesidades y gustos de los compra- } \\
\text { dores. } \\
\text { Cambios demográficos adversos. }\end{array}$ \\
\hline
\end{tabular}

Fuente: Thompson y Striklanda (1998)

Tabla 6. Matriz DOFA

\begin{tabular}{|c|c|c|}
\hline Deje en blanco & $\begin{array}{c}\text { Fortalezas-F } \\
\text { Liste las fortalezas }\end{array}$ & $\begin{array}{c}\text { Debilidades-D } \\
\text { Liste las debilidades }\end{array}$ \\
\hline $\begin{array}{c}\text { Oportunidades-O } \\
\text { Liste las oportunidades }\end{array}$ & $\begin{array}{c}\text { Estrategias FO } \\
\text { Use las fortalezas para } \\
\text { tomar ventaja de las opor- } \\
\text { tunidades }\end{array}$ & $\begin{array}{c}\text { Estrategias DO } \\
\text { Supere las debilidades } \\
\text { tomando ventaja de las } \\
\text { oportunidades }\end{array}$ \\
\hline Amenazas-A & Estrategias FA & Estrategias DA \\
\hline Liste las amenazas & $\begin{array}{l}\text { Use fortalezas para } \\
\text { evadir amenazas }\end{array}$ & $\begin{array}{c}\text { Minimice debilidades y } \\
\text { evite amenazas }\end{array}$ \\
\hline
\end{tabular}

Hecha la matriz FODA en la que se evidencian los listados de la resultante del diagnóstico en cuanto a oportunidades, amenazas, fortalezas y debilidades, se procede a la creación de la matriz MAFE como derivación de la Tabla 6. Esta matriz propuesta por David (1997) desarrolla las primeras estrategias alternativas que luego serán valoradas por la organización para su implementación y evaluación. De la matriz MAFE aparecen los siguientes modelos de estrategias: Estrategias FO, estrategias DO, estratégicas FA y estrategias DA. 
Matriz de Perfil de capacidad institucional (PCI)

Es una herramienta que se utiliza para evaluar fortalezas y debilidades en una empresa en comparación con las oportunidades y amenazas que se presentan en el medio donde desarrolla sus actividades. Es otra alternativa para realizar un diagnóstico estratégico teniendo en cuenta diferentes variables que se asocian al contexto. El PCI examina cinco categorías las cuales se mencionan a continuación: capacidad directiva, financiera, competitiva, tecnológica y de tanto humano. Serna (2008)

Tabla 7. Matriz PCI

\begin{tabular}{|c|c|c|c|c|c|c|c|c|c|c|}
\hline \multicolumn{11}{|c|}{ Perfil de la Capacidad Institucional. (P.C.l) } \\
\hline \multicolumn{11}{|c|}{ ANALISIS S INTERNO } \\
\hline & & \multicolumn{3}{|c|}{ FORTALEZAS } & \multicolumn{3}{|c|}{ DEBILIDADES } & \multicolumn{3}{|c|}{ IMPACTO } \\
\hline & & A & M & $\mathrm{B}$ & A & M & $\mathrm{B}$ & $\mathrm{A}$ & M & B \\
\hline \multirow{10}{*}{$\begin{array}{l}\text { CAPACIDAD } \\
\text { DIRECTIVA }\end{array}$} & Uso de análisis y planes estratégicos & $\mathrm{X}$ & & & & & & & $\mathrm{X}$ & \\
\hline & $\begin{array}{l}\text { Velocidad de respuesta a condiciones } \\
\text { cambiantes }\end{array}$ & & $\mathrm{X}$ & & & & & $\mathrm{X}$ & & \\
\hline & $\begin{array}{l}\text { Flexibilidad de la estructura organiza- } \\
\text { cional }\end{array}$ & & $\mathrm{X}$ & & & & & & & $\mathrm{X}$ \\
\hline & Comunicación y control organizacional & & & & $\mathrm{X}$ & & & & & $\mathrm{X}$ \\
\hline & $\begin{array}{l}\text { Experiencia y conocimiento de los direc- } \\
\text { tivos }\end{array}$ & $\mathrm{X}$ & & & & & & & $\mathrm{X}$ & \\
\hline & $\begin{array}{l}\text { Habilidad para atraer y retener indivi- } \\
\text { duos creativos }\end{array}$ & $\mathrm{X}$ & & & & & & & & $\mathrm{X}$ \\
\hline & $\begin{array}{l}\text { Habilidad para responder a tecnologías } \\
\text { cambiantes }\end{array}$ & & & & & $\mathrm{X}$ & & & & $\mathrm{X}$ \\
\hline & $\begin{array}{l}\text { Capacidad para enfrentar a la competen- } \\
\text { cia }\end{array}$ & & & $\mathbf{X}$ & & & & & & $\mathrm{X}$ \\
\hline & Sistemas de control eficaz & & & $\mathrm{X}$ & & & & & $\mathrm{X}$ & \\
\hline & Sistemas de toma de decisiones & $\mathrm{X}$ & & & & & & $\mathrm{X}$ & & \\
\hline \multirow{5}{*}{$\begin{array}{l}\text { CAPACIDAD } \\
\text { COMPETITIVA }\end{array}$} & Calidad del servicio & & $\mathrm{X}$ & & & & & & & $\mathrm{X}$ \\
\hline & Lealtad y satisfacción de los clientes & & & $\mathrm{X}$ & & & & $\mathrm{X}$ & & \\
\hline & Portafolio de servicios & & & $\mathrm{X}$ & & & & & $\mathrm{X}$ & \\
\hline & $\begin{array}{l}\text { Inversión de I+D para el desarrollo de } \\
\text { nuevos servicios }\end{array}$ & & & & & $\mathrm{X}$ & & $\mathrm{X}$ & & \\
\hline & Administración de clientes & & & & $\mathrm{X}$ & & & & & $\mathrm{X}$ \\
\hline \multirow{4}{*}{$\begin{array}{l}\text { CAPACIDAD } \\
\text { FINANCIERA }\end{array}$} & Acceso a capital cuando lo requiere & & & & & & $\mathrm{X}$ & & $\mathrm{X}$ & \\
\hline & Rentabilidad, retorno de la inversión & & & $\mathrm{X}$ & & & & & & $\mathrm{X}$ \\
\hline & Habilidad para competir con precios & & & & & $\mathrm{X}$ & & $\mathrm{X}$ & & \\
\hline & Estabilidad de costos & & $\mathrm{X}$ & & & & & & $\mathrm{X}$ & \\
\hline \multirow{3}{*}{$\begin{array}{l}\text { CAPACIDAD } \\
\text { TECNOLOGICA }\end{array}$} & Capacidad de innovación & & $\mathrm{X}$ & & & & & & $\mathrm{X}$ & \\
\hline & Nivel de tecnologías usadas & & & $\mathrm{X}$ & & & & & $\mathrm{X}$ & \\
\hline & Aplicación de tecnologías informáticas & & & & $\mathrm{X}$ & & & & & $\mathrm{X}$ \\
\hline \multirow{7}{*}{$\begin{array}{l}\text { CAPACIDAD } \\
\text { TALENTO HU- } \\
\text { MANO }\end{array}$} & Nivel académico del recurso humano & & & & & $\mathrm{X}$ & & & & $\mathrm{X}$ \\
\hline & Experiencia técnica & & & & & & $\mathrm{X}$ & & $\mathrm{X}$ & \\
\hline & Rotación interna & & & & $\mathrm{X}$ & & & & & $\mathrm{X}$ \\
\hline & Estabilidad de costos & & & $\mathrm{X}$ & & & & $\mathrm{X}$ & & \\
\hline & Ausentismo & & $\mathrm{X}$ & & & & & & $\mathrm{X}$ & \\
\hline & Pertenecía & $\mathrm{X}$ & & & & & & & & $\mathrm{X}$ \\
\hline & Nivel de remuneración & $\mathrm{X}$ & & & & & & & $\mathrm{X}$ & \\
\hline
\end{tabular}




\section{Herramientas utilizadas en prospectiva}

De acuerdo con Godet (2005) para el uso y la aplicación de la prospectiva tenemos las siguientes herramientas alternativas:

Matriz de Impactos Cruzados- Multiplicación aplicada a una clasificación: teniendo como base la información obtenida del ejercicio de diagnóstico interno y externo, se establecen relaciones por medio de matrices para determinar el grado de impacto directo o indirecto y determinar las principales variables asociadas al entorno que se estudia. Este método implica que los participantes tengan una experiencia y conocimiento demostrado en el sector objeto de análisis.
Análisis MACTOR: el uso de esta metodología se utiliza para identificar los actores en el ejercicio y los objetivos que persiguen. Los actores son los que tienen mayor grado de influencia sobre las variables claves que se obtienen del análisis estructural.

La Matriz de Alianzas y Conflictos: metodología de análisis del juego de los actores que permite tener en cuenta lo complejo de los sistemas de información, dando como resultado insumos parciales sobre el tema en cuestión. Se pretende determinar la influencia de los actores sobre las variables definidas.

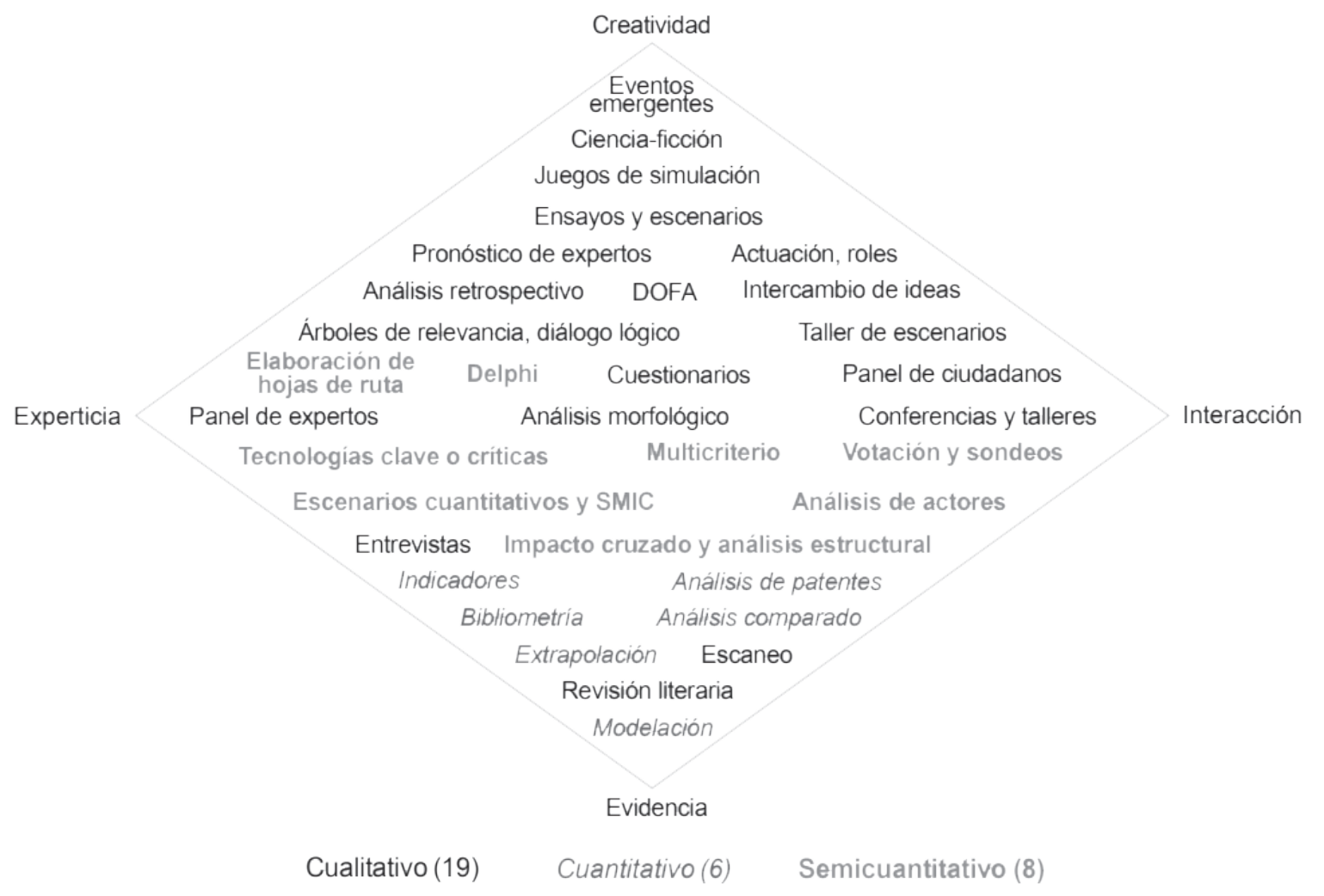

Figura 8. Diamante de métodos de la prospectiva

Fuente: El diamante de los métodos de la Prospectiva (Medina, 2006)

Análisis de los escenarios: Godet plantea que los escenarios estratégicos son la integración de compendios que conforman la imagen de una propuesta futura y la serie de hechos que permiten hacer el enlace entre el hoy (diagnóstico inicial, situación actual) y el mañana (la visión a futuro) Godet, (2000). Para Kahn y Wiener (1967) Los escenarios son lecturas narrativas del futuro que estudian los procesos causales y una serie de datos para su uso gerencial.
Hasta el momento el desarrollo de la investigación (5 PYMES como muestra piloto) han demostrado el desconocimiento de los gerentes y líderes de proceso de estas herramientas que los ayudarían a la toma de decisiones eliminando el grado de subjetividad de las mismas, dando mayor tranquilidad al equipo de trabajo generando mayor importancia al uso de datos estadísticos. 


\section{Conclusiones}

En el desarrollo de la investigación, sustento de esta ponencia plantea la importancia de las particularidades y las contribuciones teórico-prácticas sobre la utilización de la gerencia estratégica y la prospectiva, así como, las herramientas que subyacen de ella son una alternativa válida para la toma de decisiones en situaciones de incertidumbre. Se viene trabajando en el desa- rrollo de diagnósticos y escenarios utilizando las herramientas para el desarrollo metodológico del direccionamiento estratégico, la planeación estratégica y la prospectiva con las PYMES, CCC y la gobernación de Bolívar generando una línea base sirva como guía para la puesta en marcha de estrategias que le faciliten la obtención de los resultados expuestos en el plan de desarrollo y pueda generar continuidad en estos procesos de construcción social.

\section{Referencias}

Briola, M. (2007). La estrategia organizacional. Argentina: Universidad de Buenos Aires.

Codina, A. (2006). Sun Tzu y el Arte de la guerra para los negocios. Recuperado de http://www.degerencia.com/articulos.php?artid $=937$

Certo, S. (2008). Modern ManagementConcepts \& Skills. New York.Pearson Prentice Hall, 2008.

D’Alessio, F. A. (2013). El proceso estratégico. Un enfoque de gerencia. Lima, Pearson.

David, F. R. (2013). Strategic management: Concepts and cases: A competitive advantage approach. Pearson.

De jouvenel Bertrand (1964) L art de la conjeture editions du rocher. Monaco

Del Río-Cortina, A., Martín-León, M. C., Diaz-Plaza, V., \& Machado-Licona, J. (2021). Pensamiento estratégico: Una vista desde el rombo filosófico de Bédard. Saber, Ciencia y Libertad, 16(1).

Fram, T. O. B., \& Licona, J. M. (2016). La logística y la gestión de la información. Revista Cultural Unilibre, (1), 77-90.

Godet, M. (1985): Prospective et planification stratégique. Paris: Editions Economica.

Godet, M. (2000). Caja de herramientas de la prospectiva estratégica. Paris

Godet, M. (2005),"Regions facing their futures", foresight, Vol. 7 Iss: 2 pp. 21 - 27. doi: http://dx.doi.org/10.1108/14636680510590808

Godet, M. Duran (2009): Cuadernos de Lipsor. Laboratoire d'Innovation de Prospective Stratégique et d’Organisation. Colección dirigida por Marc Giget, Michel Godet e Yvon Pesqueux

Gutiérrez M (2006) los estudios y la gestión del futuro en argentina (versión electrónica) Proyecto Millenium-

Hill, C. W., Schilling, M. A., \& Jones, G. R. (2019). Administración estratégica: teoría y casos: un enfoque integral. Cengage Learning Editores.

Kahn, Herman y Wiener, Antony (1967) Toward the year 2000: a framework for speculations on the next thirty tree years. Macmillan Nueva York, Estados unidos.

Labarca, N. (2008). Evolución del pensamiento estratégico en la formación de la estrategia empresarial. Maracaibo, Venezuela: Universidad de Zulia.

Medina Vásquez, J. E., \& Ortegón, E. (2006). Manual de prospectiva y decisión estratégica: bases teóricas e instrumentos para América Latina y el Caribe. Cepal. 
Mintzberg H., Bruce Ahlstrand H., y Lampel J. (2003). Safari a la estrategia. Una visita guiada por la jungla del management estratégico. Argentina: Ediciones Granica.

Mojica F.J (2008) Forescasting y prospectiva dos alternativas complementarias para adelantarnos al futuro (versión electrónica) Colombia: Universidad externado de Colombia centro de pensamiento estratégico y prospectiva

Porter, M. E. (2008). The five competitive forces that shape strategy. Harvard business review, 86(1), 78.

Randers, J. (2012): 2052: A Global Forecast for the Next Forty Years. White River Junction: Chelsea Green Publishing

Saavedra, Juan. (2005). Administración estratégica: evolución y tendencias. Revista Economía y Administración. Extraído de: http://www2.udec.cl/ rea/REVISTA\%20PDF/Rev64/art3rea64. pdf.

Serna Gómez, H. (2008). Gerencia estratégica. Planeación y gestión, teoría y metodología. 3R Editores.

Solleiro J. \& Castañon R. (1998) Inteligencia tecnológica competitiva. Versión pragmática en revista de economía y empresa. Vol XII (2da época) (\#34)

Tarzijan, J. (2008). Fundamentos de estrategia empresarial. Elementos esenciales de la estrategia competitiva. México: Alfaomega Grupo editor S. A. 26-28

Thompson, A.y Strikland, K.F.C. (1998). Dirección y administración estratégicas. Conceptos, casos y lecturas. México: MacGraw-Hill I

Weihrich, H., y Koontz, H. (1993). Administración, una perspectiva global. México: Ed. Mc.Graw Hill. 123-191. 